\title{
Impact of Nutritional Support in Patients with Gastrointestinal Malignancies - A Review
}

\section{Dobrila-Dintinjana $\mathbf{R}^{1 *}$, Radić $\mathbf{M}^{1}$, Dintinjana $\mathbf{M}^{2}$, Redzović $\mathrm{A}^{1}$, Vukelić $\mathrm{J}^{3}, \mathbf{M}$ Zelic ${ }^{4}$, Vanis $\mathbf{N}^{5}$ and Trivanović $\mathrm{D}^{6}$}

${ }^{1}$ Radiotherapy and Oncology Clinic, University Hospital Rijeka, Croatia

${ }^{2}$ General Practice Ambulance Rijeka, Rijeka, Croatia

${ }^{3}$ Head and Neck Clinic, University Hospital Rijeka, Croatia

${ }^{4}$ Clinic for Digestive Surgery, University Hospital Rijeka, Croatia

${ }^{5}$ Gastroenterology Clinic, University Hospital Sarajevo, Bosnia and Herzegovina

${ }^{6}$ Department for Hematology and Oncology, General Hospital Pula, Croatia

\begin{abstract}
Cancer Cachexia-Anorexia Syndrome (CACS) is a common and often underdiagnosed syndrome in cancer population. If undiagnosed, this initially reversible syndrome leads to deterioration and is direct cause of death in $20 \%$ of cancer patients. Oppositely, with timely diagnosis, nutritional counseling can help to slow the progression and positively influence on quality of life, tolerance to chemotherapy with ultimate goal of prolonging patient's life. Colorectal and pancreatic cancers are very common tumors type worldwide. The prognosis for the survival in pancreatic cancer is poor as in colorectal after disease progression. Cancer anorexia-cachexia syndrome is highly prevalent among patients with colorectal and pancreatic cancer, and has a large impact on morbidity and mortality, and on patient quality of life. The etiology of primary CACS appears to be related to the pathological loss of inhibitory control of catabolic pathways, whose increased activities are not counterbalanced by the increased central and peripheral anabolic drive. Secondary CACS (related to gastrointestinal obstruction, vomiting due to chemotherapy etc.) is contributing to bad patient's condition. As a result of being complex and influencing a great number of metabolic pathways, cancer cachexia can be treated in multimodal manner. In this review we are presenting most promising targets and current opinions in ways to treat cachexia and our results with nutritional supplementation in colorectal and pancreatic cancer patients.
\end{abstract}

Keywords: Cancer Cachexia-Anorexia Syndrome (CACS); Proinflammatory cytokines; Colorectal cancer; Pancreatic cancer; Nutritional supplementation

\section{Introduction}

Cachexia is a clinical syndrome of a distressing and debilitating condition, affecting significant numbers of patients with advanced malignant disease and causing huge distress; it is the primary cause of death in about $20 \%$ of all patients with cancer. Although cachexia is most commonly associated with particular tumours, such as head and neck, gastrointestinal tract, pancreas, central nervous system and lungs, it may affect any patient with any tumour at any site [1].

Cancer anorexia-cachexia syndrome (CACS) [2,3], usually consists of a combination of anorexia, tissue wasting, malnutrition, weight loss and loss of compensatory increase in feeding. Anorexia represents the result of a failure of the usual appetite signals and is preceding cachexia (pre-cachexia). Biochemistry tests in cachectic patients are expressing anaemia, hypoalbuminemia, hypertriglyceridemia and glucose intolerance due to insulin resistance.

Primary cachexia represents the result of a complex interaction between cancer growth and host response, and is associated with a poor response to chemotherapy with an increase in drug-related toxicity [4].

Other debilitating conditions which decrease food intake (gastrointestinal obstruction, nausea and vomiting due to chemo and radiotherapy, pain, emotional factors, renal impairment etc.) are causing secondary cachexia.

Weight loss can be unrelated to reduced nutritional intake and is mostly caused by elevated resting energy expenditures (REE) and proinflammatory cytokine expression [5].

One important mechanism is the activation of the acute phase response cascade [4]. Kemik et al. found significantly higher serum CRP, IL-1 $\alpha$, IL-1 $\beta$, IL-6, IL-8, IL-10, TNF- $\alpha$, VEGF-A, VEGF-C,
VEGFR1, and leptin concentrations in patients with esophageal, gastric, pancreas, colon, and rectum cancers than controls and lower levels of the serum albumin, midkine, adiponectin, and ghrelin in patients with esophageal, gastric, pancreas, colon, and rectum cancers compared to control subjects [6].

Proinflammatory responses are stimulating the expression and release of leptin. This hormone, secreted by adipose tissue, plays a crucial role in the homeostasis of body weight as its high levels in the brain decrease the activity of the hypothalamic orexigenic mediators (ghrelin, neuropeptide Y, agoutin, orexin, melanocortin-releasing hormone) and increase anorexigenic signals (cholecystokinin, glucagon-like peptide, pro-opiomelanocortin, thyroid-releasing hormone, corticotropin-releasing hormone, oxytocin). Leptin levels regulate rest energy expenditure (REE) [7].

A range of novel drug targets relevant to the treatment of cachexia are discovered. Interventions may be either upstream (e.g. by antagonizing key mediators of systemic inflammation) or downstream (e.g. by blocking catabolic pathways or stimulating anabolic pathways in skeletal muscle). Upstream targets have the advantage of affecting multiple aspects of cachexia. For example, interleukin-6 is known to be the main mediator of the hepatic acute phase response in humans, but it may also play a role in anorexia, fatigue, anaemia, oedema and

*Corresponding author: Dobrila-Dintinjana R, Radiotherapy and Oncology Clinic University Hospital Rijeka, Croatia, E-mail: renatadobrila@windowslive.com

Received November 20, 2012; Accepted December 15, 2012; Published December 17, 2012

Citation: Dobrila-Dintinjana R, Radić M, Dintinjana M, Redzović A, Vukelić J, et al (2013) Impact of Nutritional Support in Patients with Gastrointestinal Malignancies - A Review. J Nutr Disorders Ther S11: 001. doi:10.4172/2161-0509.S11-001

Copyright: (C) 2013 Dobrila-Dintinjana R, et al. This is an open-access article distributed under the terms of the Creative Commons Attribution License, which permits unrestricted use, distribution, and reproduction in any medium, provided the original author and source are credited. 
muscle loss. By contrast, myostatin acts as a physiological brake to the continued growth of skeletal muscle and is therefore a potential downstream target. Alternative targets include the melanocortin pathway in the CNS control of appetite. Therapies based on these pathways are currently in Phase I and Phase II clinical trials in cancer patients [8].

Progestogens, particularly megestrol acetate, are commonly used to treat CACS. The mechanism of action of megestrol is believed to involve the stimulation of appetite by both direct (neuropeptide $\mathrm{Y}$ ) and indirect pathways.

Omega-3 polyunsaturated fatty acids have been shown to modulate levels of proinflammatory cytokines, hepatic acute-phase proteins, eicosanoids, and tumor-derived factors in animal models of cancer and may reverse some aspects of the process of cachexia. The metabolites of EPA and DHA have less inflammatory and immunosuppressant potency than the substances derived from arachidonic acid. The competitive metabolism of EPA and DHA with arachidonic acid is ocurring at the cyclooxygenase and 5-lipoxygenase levels [7]. Oral EPA has been found to stabilize the body weight of cancer patients and together with an energy- and protein-rich nutritional supplement, can enchance weight gain by increasing lean body mass.

Some other drugs also showed positive influence in CACS. Thalidomide, which is an inhibitor of tumor necrosis factor-alpha synthesis, may represent a rational therapeutic approach [9] also as inexpensive oral supplementation of L-Carnitin [10].

In postoperative setting, early nutritional support was shown to reduce the incidence of complications and to shorten the hospital stay. For patients with functional bowel, early enteral nutrition (EEN) is method of choice, and total parenteral nutrition (TPN) is reserved for highly selected cases $[11,12]$. If patient's condition cannot provide normal feeding (but gastrointestinal tract is functional) or anticipated time for feeding is longer than 4 weeks, trans nasal way can be used (nasogastric, nasoduodenal and nasojejunal tubes). Percutaneous endoscopic gastrostomy tubes or percutaneous endoscopic jejunostomy may be placed (endoscopic or surgical way) if longer period of feeding is anticipated.

Pancreatic and colorectal cancer are most common gastrointestinal malignancies. Pancreatic cancer (PCa) is a very aggressive, invasive cancer whose prognosis remains very poor and represents the fourth leading cause of cancer-related mortality. Only $5 \%$ of patients is living longer than 5 years. There are approximately 277,000 new cases of pancreatic cancer and 266,000 deaths from pancreatic cancer annually in Europe, indicating a mortality rate of $96 \%$ of the cases diagnosed. Etiology of pancreatic carcinoma remains largely unknown but consistent evidence of a positive association was found for family history and cigarette smoking. Also, some studies showed a positive association with diabetes mellitus and chronic pancreatitis.

Cancers of the colon and rectum (CRC) are the third most common forms of cancer worldwide. In the developed countries CRC is the significant cause of morbidity and mortality (412 000 new diagnosed patients in Europe every year). The overall five-year survival for colon cancer in varied from $43 \%$ (Europe) to $62 \%$ (USA) [13-20].

There are four risk factor categories for CRC: epidemiological, intestinal, dietetic, and mixed. CRC is a disease in which genetic mutations of somatic cells are the molecular base of the disease. About $25-30 \%$ of CRC are diagnosed in the advanced stage and another $30 \%$ of patient will develop metastatic or locally advanced disease in next three years. Despite advances in therapeutic methods, the five-year survival rate for advanced disease is still poor (15\%).
For patients with advanced stage of CRC and PCa, the therapeutic goal is quality of life (QoL). Early intervention with nutritional supplementation has been shown to halt malnutrition, reduce the consequences of CACS, extend patient survival and improve quality of life.

\section{Aim of our Studies}

In our studies we tried to assess the influence of nutritional support (counseling, enteral supplementation liquids, megestrol acetate) on nutritional status and symptoms prevalence in patients with pancreatic and colorectal cancer during chemotherapy.

\section{Methods and Patients}

During our routine clinical work nutritional status of our patients is evaluating according to changes in body weight. Body Mass Index (BMI) is calculated for all patients (on chemo/biotherapy procedures or achieving nutritional support) using the standard procedure of dividing weight in $\mathrm{kg}$ by height in $\mathrm{m}^{2}$. We also use following questionnaires for evaluation of patient's nutritional status: Nottingham Screening Tool Score (NST score 0-7) (Table 1), Appetite Loss Scale (0-10; where 0 is no appetite at all, and 10 is the best possible appetite), and for Performance Status or Eastern Cooperative Oncology Group (ECOG) (Table 2) or Karnofsky Performance Status (KPS) (Table 3).

Nutritional and pharmacological support is consisting of nutritional counseling, and if according to NST patients are in nutritive risk, administration of $10 \mathrm{ml}(400 \mathrm{mg}$ ) per day Megostat (megestrol acetate) and enteral nutrition supplements with commercially available products.

Nutritional counseling include interviews with a physician, with purpose of learning how to prepare and ingest food during chemotherapy and to change eventually bad eating habits.

We analysed impact of nutritional support in patients with histologically confirmed pancreatic cancer (PCa) patients during 18 months period (from $1^{\text {st }}$ July 2005 to $31^{\text {st }}$ December 2006 at Gastroenterology department, Clinical Hospital Centre Rijeka). We followed up 44 patients with pancreatic cancer - 26 males (mean age 69 years \pm 2.4 years) and 18 females (mean age $63 \pm 3.2$ years). All patients were with metastatic or locally advanced disease curing with standard

\section{BMI}

$$
\begin{aligned}
& 0-\mathrm{BMI}>20 \\
& 1-\mathrm{BMI} 18-20 \\
& 2-\mathrm{BMI}<18
\end{aligned}
$$

Has the patient unintentionally lost weight during last 3 months?

$0-$ no

1 - a little, up to $3 \mathrm{~kg}$

2 - a lot, more than $3 \mathrm{~kg}$

Food intake - has this increase during the last month prior the estimation?

$0-0$

$1-$ yes

Stress factor/severity of illness?

0 - none

1 - moderate (uncomplicated operation, chronic disease, infection, etc.

2 - severe (multiple fractures and wounds, sepsis, cancers, major operation, etc) Score 0-2 Patient do not need nutritive support

Score 3-4 Patient had to be monitored once more in week

Score 4-5 Patient has malnutrition and need nutritive counseling and support

BMI-Body Mass index

Table 1: Nothingam screening tool questionnaire. 


\begin{tabular}{|l|l|}
\hline Grade & ECOG \\
\hline 0 & Fully active, able to carry on all pre-disease performance without restriction \\
\hline 1 & Restricted in physically strenuous activity but ambulatory and able to carry out work of a light or sedentary nature, e.g., light house work, office work \\
\hline 2 & Ambulatory and capable of all selfcare but unable to carry out any work activities. Up and about more than $50 \%$ of waking hours \\
\hline 3 & Capable of only limited selfcare, confined to bed or chair more than $50 \%$ of waking hours \\
\hline 4 & Completely disabled. Cannot carry on any selfcare. Totally confined to bed or chair \\
\hline 5 & Dead \\
\hline
\end{tabular}

Table 2: Eastern Cooperative Oncology Group scoring scale of performance status.

\begin{tabular}{|l|l|}
\hline Value & Level of Functional Capacity \\
\hline 100 & Normal, no complaints, no evidence of disease \\
\hline 90 & Able to carry on normal activity, minor signs or symptoms of disease \\
\hline 80 & Normal activity with effort, some signs or symptoms of disease \\
\hline 70 & Cares for self, unable to carry on normal activity or to do active work \\
\hline 60 & Requires occasional assistance, but is able to care for most needs \\
\hline 50 & Requires considerable assistance and frequent medical care \\
\hline 40 & Disabled, requires special care and assistance \\
\hline 30 & Severely disabled, hospitalization is indicated although death is not imminent \\
\hline 20 & Hospitalization is necessary, very sick, active supportive treatment necessary \\
\hline 10 & Moribund, fatal processes progressing rapidly \\
\hline 0 & Dead \\
\hline
\end{tabular}

Table 3: Karnofsky performance scale.

chemotherapeutic protocol (gemcitabine $1000 \mathrm{mg} / \mathrm{m}^{2}$ weekly for seven consecutive weeks) [21].

We analysed colorectal cancer (CRC) patients in study which took place in the Gastroenterology and Oncology Department, Clinical Hospital Centre Rijeka and the Oncology Department, General Hospital Pula, from January 2000 to December 2009. All six hundred and twenty eight (628) patients had initial diagnosis of locally advanced and/or metastatic colorectal adenocarcinoma which was confirmed histologically and were referred for first-line standardized chemo/ biotherapy combination regimen of Folfiri/Xeliri or Folfox/Xelox + bevacizumab. We divided this proportion of patients in two groups and compared them: group I (315 patients) was monitored prospectively and achieved nutritional support and group II (313 patients) from whom the data were collected retrospectively over a five-year time period (when nutritional supplements were not available in Croatia). The median age of patients in Group I was $68 \pm 2.9$ years, and $67 \pm 2.7$ in Group II.

In all patients we used the same procedure for establish nutritional status as already described above. Nutritional and pharmacological support consisted of nutritional counseling, administration of $10 \mathrm{ml}$ (400 mg) per day Megostat (megestrol acetate) and enteral nutrition supplements with commercially available products Ensure $(400 \mathrm{~mL}$ daily with $600 \mathrm{kcal}$ ) and Prosure product containing eicosapentaenoic acid (EPA) (480 ml daily, containing $2.2 \mathrm{~g}$ of EPA and $600 \mathrm{kcal})$. In the beginning of observation only this products were available in Croatia so we continue to use it during whole period of study.

Both, PCa patients and CRC patients had first visit 0-4 weeks after surgery if performed or one week before chemotherapy initiation. Patients were counseled and re-evaluated by the same physician before the start of chemotherapy, and every two weeks during 12 visits (CRC patients) or 5 visits (PCa patients) in connection with scheduled visits to the oncology unit.

By using nutritional counseling, enteral food substitution and pharmacological support PCa patients stopped to lose weight. This effect was most pronounced 4-6 weeks after beginning the supportive therapy, in fact, $36(81.8 \%)$ patients increased weight. Median weight gain was $2.5 \mathrm{~kg}$ ( 1.8 to $4.5 \mathrm{~kg}$ ). Appetite range on numeric rate scale increases from 2.7 at baseline up to 4.5 (Table 4 ).

Diarrhea in $11.4 \%$ and abdominal pain in $15.9 \%$ were the only side effects of enteral liquid supplementation and from megestrol acetate edemas in $36.4 \%$ patients.

In study with CRC patients at initial visit, upon evaluating the risk measurements according to BMI, decrease in weight, and NST, we did not find any significant differences between the prospective and retrospective group. After completion of chemotherapy and nutritional support cycle, comparing these two groups we noticed weight gain in those with a BMI $<20$ who received counseling and nutrition and an opposite effect was observed in the group without nutritional support. After 4 weeks of supplementation in Group I, 73.34\% patients had an increase in body weight, with an average weight gain of $1.5 \mathrm{~kg}(0.6-3.3$ $\mathrm{kg}$ ) versus Group II where increase in weight gain was observed in only $19.49 \%$ of patients. Patients who achieved nutritional supplementation also expressed appetite improvement from 3.3 to 4.6 on Appetite Loss Scale. On week 12 there was a significantly smaller proprotion of patients with $\mathrm{BMI}<20$ and NST $\geq 5$ in the group with nutritional counseling. A greater proportion of patients in the same group had a better appetite according to Appetite Loss Scale (Table 5).

As side effects patients in Group I comparing with patients in Group II expressed more diarrhea $17.5 \%$ vs. $13 \%$ of patients and more abdominal pain in $15.9 \%$ vs. $12 \%$ of patients. Edemas, the main side effect of megestrol acetate was experienced in $29.2 \%$ of patients.

We also analyzed survival in the two groups and we determine that patients with nutritional support had a significantly longer median survival than patients in the control group (19.1 vs. 12.4 months).

\section{Discussion}

At the time of diagnosis patients with PCa mostly have disseminated disease; $20 \%$ of patients who does not have macroscopic disseminated disease have positive intra-abdominal lymph nodes when using Polymerase Chain Reaction (PCR) method. Therefore, despite the clear advances in surgical treatment of pancreatic carcinoma, 5 years survival rates are still low, ranging from 5-30\%. Early metastasizing and 


\begin{tabular}{|l|l|l|l|l|l|l|}
\hline \multirow{2}{*}{ Visit } & Patients & \multirow{2}{*}{ KPS } & \multicolumn{2}{l|}{ Weight Gain $>\mathbf{~ k g}$} & Apetite Numeric Range Scale (0-10) \\
\cline { 2 - 3 } & number & $\%$ & number & $\%$ & $0.0 \%$ & 2.7 \\
\hline 0 & 44 & $100.0 \%$ & 70.4 & 0 & $72.7 \%$ & 4.2 \\
\hline 1 & 44 & $100.0 \%$ & 73.6 & 32 & $81.8 \%$ & 4.2 \\
\hline 2 & 44 & $100.0 \%$ & 78.0 & 36 & $79.0 \%$ & 4.5 \\
\hline 3 & 38 & $86.4 \%$ & 76.6 & 30 & $61.1 \%$ & 4.2 \\
\hline 4 & 36 & $81.8 \%$ & 70.4 & 22 & \\
\hline
\end{tabular}

Table 4: Karnofsky performance status, weight increasing and appetite numeric range scale in follow up of patients with pca.

\begin{tabular}{|c|c|c|c|c|}
\hline & \multicolumn{2}{|c|}{ Group I (with nutritional counseling) } & \multicolumn{2}{|c|}{ Group II (without nutritional counseling) } \\
\hline & Visit 0 & Visit 12 & Visit 0 & Visit 12 \\
\hline $\mathrm{BMI}<20$ & $\begin{array}{l}157 \\
(49.84 \%)\end{array}$ & $\begin{array}{l}118 \\
(37.46 \%)\end{array}$ & $\begin{array}{l}152 \\
(48.57 \%)\end{array}$ & $\begin{array}{l}201 \\
(64.22 \%)\end{array}$ \\
\hline NST $\geq 5$ & $\begin{array}{l}155 \\
(49.21 \%)\end{array}$ & $\begin{array}{l}107 \\
(33.97 \%)\end{array}$ & $\begin{array}{l}149 \\
(47.60 \%)\end{array}$ & $\begin{array}{l}194 \\
(61.99 \%)\end{array}$ \\
\hline loss of appetite & $\begin{array}{l}246 \\
(78.10 \%)\end{array}$ & $\begin{array}{l}110 \\
(34.92 \%)\end{array}$ & $\begin{array}{l}223 \\
(71.25 \%)\end{array}$ & $\begin{array}{l}249 \\
(79.05 \%)\end{array}$ \\
\hline $\begin{array}{l}\text { Decrease in weight gain (>2 } \\
\mathrm{kg} / \text { month) }\end{array}$ & $\begin{array}{l}143 \\
(45.40 \%)\end{array}$ & $\begin{array}{l}88 \\
(27.94 \%)\end{array}$ & $\begin{array}{l}137 \\
(43.77 \%)\end{array}$ & $\begin{array}{l}252 \\
(80.51 \%)\end{array}$ \\
\hline
\end{tabular}

NST: Notthingam Screening Tool

BMI: Body Mass Index

Table 5: Number of patients in Group I and Group II with BMI $<20$, NST $\geq 5$, loss of apetite and decrease in weight gain ( $>2 \mathrm{~kg} / \mathrm{month}$ ).

low sensitivity to anticancer treatment modalities (due to biological phenotype of $\mathrm{PCa}$ ), are responsible that mortality rate is the highest in all types of cancer.

PCa patients are suffering from appetite loss and they are decreasing in weight gain due to multicausal reasons: slow postoperative recovery, depression, restricted dietary intake, emesis, early satiety, abdominal pain following meal. PCa patients also have continuous suppression of appetite and increase of energy expenditure due to increased level of leptin.

More than $80 \%$ of PCa cases develop CACS; and that it is among highest incidence of CACS compared to other tumors. Therefore the $\mathrm{PCa}$ is paradigm for investigation and treatment of CACS.

CRC is second in global cancer incidence with increased risk in industrialized nations. There is no incidence difference between genders for CRC which is the most common cause of cancer death among non-smokers. Despite vast achievements in surgery, chemo/bio and radiotherapy, the percentage of 5 -year-survivals is still poor and reaches $15 \%$.

CRC is a heterogeneous disease which is developing through complex series of molecular changes which are only partly known. Substantial proportion of CRC patients develops CACS. Depending of disease spreading, antitumor treatments and its complications, we can find CACS in $30-50 \%$ of CRC patients.

There is no single, generally agreed-upon definition of cachexia [12]. Therefore, cachexia is infrequently identified, and rarely treated [12]. It is essential to have a validated and universally accepted definition of cachexia so clinicians can recognize the problem and institute treatment $[12,22]$.

The loss of muscle and fat tissue is mediated by different alterations in the organism, not all of which are known until now [23].

Pro-inflammatory cytokines, (TNF-alpha, IL-1beta, IL-6, IL-8), enhanced the expression of Leukemia inhibitory factor- pleiotropic cytokine (LIF) which significantly induced cell proliferation [17]. Acute-phase response proteins are related to cachexia, accelerated angiogenesis in gastroesophageal cancers and decreased survival [24].
Ghrelin, a 28-amino acid peptide, also express a proliferative effect in neoplastic disorders [25].

Muscle wasting in cancer patients is consequence from activation of the ubiquitin proteasome pathway by proteolysis inducing factor (PIF) which is independent of nutrient intake, and therefore nutritional supplementation alone is unable to reverse the process of cachexia. EPA prevents muscle wasting by down-regulating the increased expression of this pathway [26].

After a median of at least 3-month supplementation with EPA, positive changes in weight, significant reduction of acute-phase protein production and the stabilization of resting energy expenditure were registered in many studies $[27,28]$. Studies suggest that analyzed progestational agents (megestrol acetate and medroxyprogesterone), showed that those agents improve appetite and increase weight in advanced cancer patients with slight increase in risk of thrombophlebitis. Also, eicosapentaenoic acid (EPA) in phases I and II produce a decrease in proinflammatory interleukins and TNFa and open a possibility of reverse cancer cachexia in early stages.

Although reported results are inconclusive in studies phase III, in every-day clinical practice, faced with patients with CACS (or with possibility to develop CACS) we are offering nutritional counseling and nutritional support with EPA and megestrol acetate to our patients with satisfactory clinical outcome. Accordingly to previous reports, our patients also tolerated enteral food supplementation and megestrol acetate well; only a few percents of them experienced diarrhea which followed abdominal pain due to enteral liquid supplementation. Megestrol acetate induced edemas in relatively significant proportion of patients (36.4\% and $29.2 \%$ respectively) but patients doesn't forfeit from this medication. Improved social life due to the better appetite is more important than discomfort caused by edema. Despite the improvement in weight and appetite, Performance Status of cancer patients isn't changing significantly trough period of nutritional supplementation, reflecting the seriousness of illness itself.

One problem of CACS is how to implement (and which Tool) for early detection of precachexia in clinical practice. Pre-cachexia is found to be condition preceeding cachexia and it's characterized with progressive wasting of skeletal muscle mass and it's replacement with 
adipose tissue before weight loss. There is general failure to recognize patients in nutritive risk early enough and to implement effective nutrition interventions.

Multimodal approach (megestrol acetate, EPA, L-carnitine and thalidomide) has shown better treatment outcome than giving single preparation [29]. Thalidomide also causes increased appetite and body weight, but at a dose of $200 \mathrm{mg}$ caused severe side effects, which make his use controversial. Nonsteroidal anti-inflamatory drugs (NSAIDs) are also interesting in their application to cancer cachexia because they can reduce systemic inflammation. Open-label studies with celecoxib and L-carnitine in preliminary experiments have shown an increase in body weight.

The infusion of ghrelin in patients with breast cancer increased appetite, food intake and feeding satisfaction, with no apparent side effects. Oral ghrelin mimetics increase body mass [30-32]. But as ghrelin and its mimetics have the ability to cause increased levels of IGF-1, it is necessary to examine the safety of their use in larger clinical studies.

Nonsteroid selective androgen receptor modulators (SARM) can havea positive anabolic effect while avoiding virilization and hypertrophy of the prostate gland in men. Application of SARM in postmenopausal women and older men with a tumor cachexia showed weight gain and better functioning of patients with relatively few side effects [33]. In studies of phases I and II by Bayliss et al. it was applied a monoclonal antibody to IL-6 in patients with NSCLC. The results showed improvement in body weight and reduced disease symptoms level [34].

As some other authors, we concluded that nutritional counseling and nutritional support can temporarily stop weight loss and improve appetite, social life and quality of life in gastrointestinal cancer patients. In study with CRC patients we even demonstrated impact on survival rate. However, due to a relatively small number of patients and short follow up, those results has to be considered with great caution.

Today we have gap between necessity of preventing and treating CACS and paucity of clear recommendations and evidence for supportive nutritional therapy for oncology patients. We have ethical problems of supplemental nutrition in patients with advanced tumor illness and also problems of cost-benefit ratio of nutritional supplementation in view of cost development in the public health system. Although we can expect new drugs from outgoing studies, it will take a time for their implementation in clinical practice. Symptom control, counseling on nutrition and appropriate physical activity levels are still base for the good treatment of cancer cachexia. Other anabolic preparations such as ghrelin, thalidomide, SARM and monoclonal antibodies require further clinical testing in order to distinguish their performance, but they represent a new frontier in the multimodal treatment of cancer cachexia. Although we can expect new drugs from outgoing studies, it will take a time for their implementation in clinical practice

Therefore, we still need more phase III studies of already existing nutrition supplements with large number of patients. We can conclude that the role of nutrition therapy is still assumed to be less important than tumor response on antitumor therapy as outcomes are less clear in literature.

No single preparation can be considered as a standard in the treatment of cancer cachexia. Currently, in the treatment of cancer cachexia, are well-established preparations containing EPA, megestrol acetate and corticosteroids.

\section{References}

1. Holmes S (2009) A difficult clinical problem: diagnosis, impact and clinical management of cachexia in palliative care. Int J Palliat Nurs 15: 320, 322-326.

2. Inui A (2002) Cancer anorexia-cachexia syndrome: current issues in research and management. CA Cancer J Clin 52: 72-91.

3. Bruera E, Sweeney C (2000) Cachexia and asthenia in cancer patients. Lancet Oncol 1: 138-147.

4. Bachmann J, Ketterer K, Marsch C, Fechtner K, Krakowski-Roosen H, et al. (2009) Pancreatic cancer related cachexia: influence on metabolism and correlation to weight loss and pulmonary function. BMC Cancer 9: 255.

5. Ravasco P, Monteiro-Grillo I, Camilo M (2007) Colorectal cancer: intrinsic characteristics modulate cancer energy expenditure and the risk of cachexia. Cancer Invest 25: 308-314.

6. Kemik O, Kemik AS, Begenik H, Erdur FM, Emre H, et al. (2012) The relationship among acute-phase responce proteins, cytokines, and hormones in various gastrointestinal cancer types patients with cachectic. Hum Exp Toxicol 31: 117-125.

7. Uomo G, Gallucci F, Rabitti PG (2006) Anorexia-cachexia syndrome in pancreatic cancer: recent development in research and management. JOP 7 157-162.

8. Fearon KC, Baracos VE (2010) Cachexia in pancreatic cancer: new treatment options and measures of success. HPB (Oxford) 12: 323-324.

9. Gordon JN, Trebble TM, Ellis RD, Duncan HD, Johns T, et al. (2005) Thalidomide in the treatment of cancer cachexia: a randomised placebo controlled trial. Gut 54: 540-545.

10. Kraft M, Kraft K, Gärtner S, Mayerle J, Simon P, et al. (2012) L-Carnitinesupplementation in advanced pancreatic cancer (CARPAN)--a randomized multicentre trial. Nutr J 11: 52.

11. Fearon KC, Barber MD, Falconer JS, McMillan DC, Ross JA, et al. (1999) Pancreatic cancer as a model: inflammatory mediators, acute-phase response, and cancer cachexia. World J Surg 23: 584-588.

12. Evans WJ, Morley JE, Argilés J, Bales C, Baracos V, et al. (2008) Cachexia: a new definition. Clin Nutr 27: 793-799.

13. Granata R, Isgaard J, Alloatti G, Ghigo E (2011) Cardiovascular actions of the ghrelin gene-derived peptides and growth hormone-releasing hormone. Exp Biol Med (Maywood) 236: 505-514.

14. Fearon KC, Voss AC, Hustead DS; Cancer Cachexia Study Group (2006) Definition of cancer cachexia: effect of weight loss, reduced food intake, and systemic inflammation on functional status and prognosis. Am J Clin Nutr 83 1345-1350.

15. Beloueche-Babari M, Chung YL, Al-Saffar NMS, Falck-Miniotis M, Leach MO (2010) Metabolic assessment of the action of targeted cancer therapeutics using magnetic resonance spectroscopy. $\mathrm{Br} \mathrm{J}$ Cancer 102: 1-7.

16. Zoheir Y, Leroux C, Picot D, Boucher E, Audrain O, et al. (2010) Changes in resting energy expenditure (REE) as a function of tumor response to sunitinib in a cachexic patient with a metastatic endocrine tumor of the pancreas. Acta Oncol 49: 1390-1391.

17. Kamohara H, Ogawa M, Ishiko T, Sakamoto K, Baba H (2007) Leukemia inhibitory factor functions as a growth factor in pancreas carcinoma cells: Involvement of regulation of LIF and its receptor expression. Int J Oncol 30: 977-983.

18. Schmitt TL, Martignoni ME, Bachmann J, Fechtner K, Friess $\mathrm{H}$, et al. (2007) Activity of the Akt-dependent anabolic and catabolic pathways in muscle and liver samples in cancer-related cachexia. J Mol Med (Berl) 85: 647-654.

19. Martignoni ME, Dimitriu C, Bachmann J, Krakowski-Rosen H, Ketterer K, et al (2009) Liver macrophages contribute to pancreatic cancer-related cachexia. Oncol Rep 21: 363-369.

20. Moses AG, Maingay J, Sangster K, Fearon KC, Ross JA (2009) Proinflammatory cytokine release by peripheral blood mononuclear cells from patients with advanced pancreatic cancer: relationship to acute phase response and survival. Oncol Rep 2: 1091-1095.

21. Dintinjana RD, Guina T, Krznaric Z, Radic M, Dintinjana M (2008) Effects of nutritional support in patients with colorectal cancer during chemotherapy. Coll Antropol 32: 737-740. 
Citation: Dobrila-Dintinjana R, Radić M, Dintinjana M, Redzović A, Vukelić J, et al. (2013) Impact of Nutritional Support in Patients with Gastrointestinal Malignancies - A Review. J Nutr Disorders Ther S11: 001. doi:10.4172/2161-0509.S11-001

22. Fox KM, Brooks JM, Gandra SR, Markus R, Chiou CF (2009) Estimation of Cachexia among Cancer Patients Based on Four Definitions. J Oncol 2009: 693458.

23. Costelli P, Baccino FM (2003) Mechanisms of skeletal muscle depletion in wasting syndromes: role of ATP-ubiquitin-dependent proteolysis. Curr Opin Clin Nutr Metab Care 6: 407-412.

24. Krzystek-Korpacka M, Matusiewicz M, Diakowska D, Grabowski K, Blachut $\mathrm{K}$, et al. (2008) Acute-phase response proteins are related to cachexia and accelerated angiogenesis in gastroesophageal cancers. Clin Chem Lab Med 46: 359-364.

25. Duxbury MS, Waseem T, Ito H, Robinson MK, Zinner MJ, et al. (2009) Ghrelin promotes pancreatic adenocarcinoma cellular proliferation and invasiveness. Biochem Biophys Res Commun 309: 464-468.

26. Tisdale MJ (2003) The 'cancer cachectic factor'. Support Care Cancer 11: 7378.

27. Barber MD, Ross JA, Voss AC, Tisdale MJ, Fearon KC (1999) The effect of an oral nutritional supplement enriched with fish oil on weight-loss in patients with pancreatic cancer. Br J Cancer 81: 80-86.

28. Barber MD, Fearon KC, Tisdale MJ, McMillan DC, Ross JA (2001) Effect of a fish oil-enriched nutritional supplement on metabolic mediators in patients with pancreatic cancer cachexia. Nutr Cancer 40: 118-124.
29. Mantovani G, Macciò A, Madeddu C, Serpe R, Massa E, et al. (2010) Randomized phase III clinical trial of five different arms of treatment in 332 patients with cancer cachexia. Oncologist 15: 200-211.

30. Neary NM, Small CJ, Wren AM, Lee JL, Druce MR, et al. (2004) Ghrelin increases energy intake in cancer patients with impaired appetite: acute, randomized, placebo-controlled trial. J Clin Endocrinol Metab 89: 2832-2836.

31. Garcia JM, Polvino WJ (2007) Effect on body weight and safety of RC-1291, a novel, orally available ghrelin mimetic and growth hormone secretagogue: results of a phase I, randomized, placebo-controlled, multiple-dose study in healthy volunteers. Oncologist 12: 594-600.

32. Garcia J, Boccia RV, Graham C, Kumor K, Polvino W (2007) A phase I randomized, placebo-controlled, double-blind study of the efficacy and safety of RC-1291 (RC) for the treatment of cancer cachexia. J Clin Oncol 25: 525S.

33. Mohler ML, Bohl CE, Jones A, Coss CC, Narayanan R, et al. (2009) Nonsteroidal selective androgen receptor modulators (SARMs): dissociating the anabolic and androgenic activities of the androgen receptor for therapeutic benefit. J Med Chem 52: 3597-3617.

34. Bayliss TJ, Smith JT, Schuster M, Dragnev KH, Rigas JR (2011) A humanized anti-IL-6 antibody (ALD518) in non-small cell lung cancer. Expert Opin Bio Ther 11: 1663-1668 